\title{
Law of Universal Gravitation \& The Cosmology
}

\section{Huai Tang Gu*}

The middle school of Dong-Xin Farm, lianyungang city, jiangsu province, China

*Corresponding author details: Huai Tang Gu; ght19651213@163.com

\begin{abstract}
For a long time, because of the complexity of galactic structure and the multiplicity of celestial body motion, the law of universal gravitation has not been applied to a galaxy and the universe as a whole. By using the method of systems analysis, a galaxy is studied as a whole or a mass point that the law of universal gravitation is successfully applied, and obtain the universe is a large material system, which can be divided into many small sub-systems, and the sub-systems can be divided into many minor grandson systems, thus infinitely divided until the lowest level of the universe, that is, planets. Regardless of the level, all galaxies exist in the form of a sphere consisting of many secondary galaxies and their orbits, and this sphere moves regularly in a similar circular elliptical orbit. The kinetic energy and potential energy are the existing basic forms of energy in the universe, and the internal energy is a complementary existing form of energy under relativity rest condition. Because of the multiple motion of the universe, the kinetic energy in the universe exists on the division of energy levels, the higher the levels of the galaxy structure are, the lower the kinetic energy levels are, and the lower the levels of a galaxy structure are, the higher its kinetic energy levels are. Although there is an evolution of mass and energy distribution in the universal system, but it is eternal for the whole universal system.
\end{abstract}

Keywords: cosmology: theory; celestial mechanics; galaxies: structure; Sun: activity; chaos

\section{INTRODUCTION}

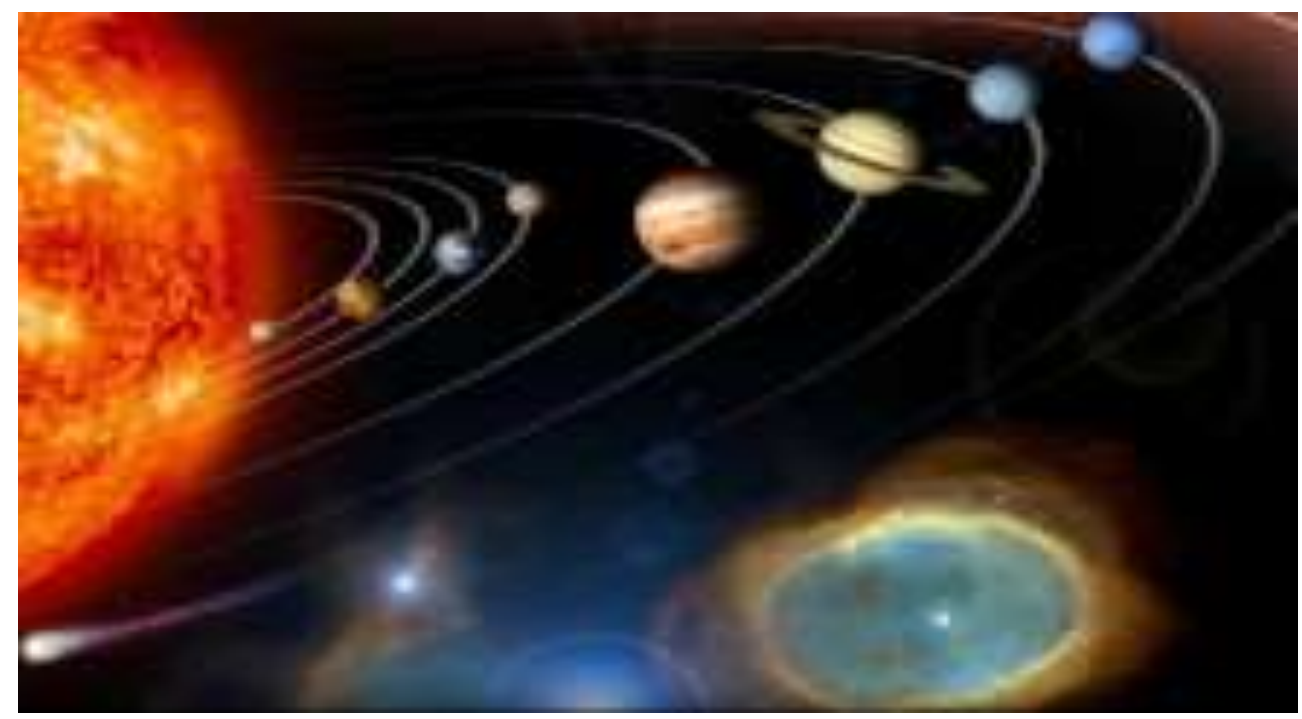

FIGURE 1: The structure of the Solar System

\section{A reverie produced from the structure of the Solar System}

It is well known the Moon is centered on the Earth and moves around the Earth in a fixed elliptical orbit to form the Earth system; the Earth, together with other planets, are centered on the Sun and move around the Sun in respective elliptical orbits to form the Solar System, thus it can be inferred the Solar System together with other same level galaxies is centered on the Galactic Center and move around the Galactic Center in their respective elliptical orbits to form the Milky Way; The Milky Way is together with other same level galaxies to form the upper level galaxy of the Milky Way, that is, a Local Group of Galaxies (Chen Gong fu, 2000). And can deduce the rest from this, to make up the whole universe. 


\section{METHOD}

(1) Part of mass:

Law of universal gravitation \& the cosmology

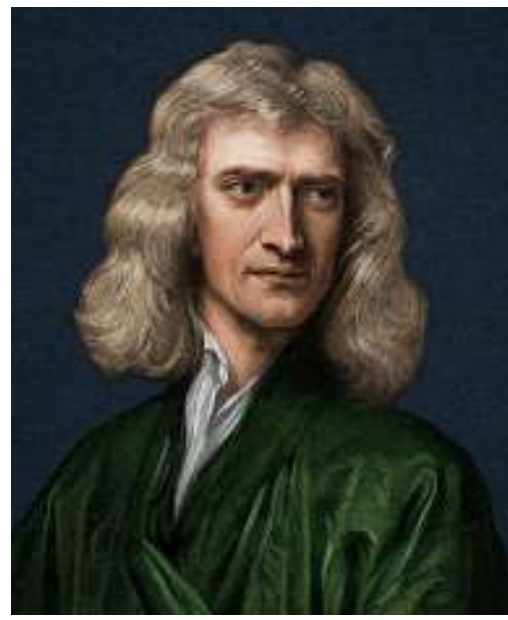

FIGURE 2: Law of universal gravitation's finder

In theory of cosmology, the law of universal gravitation is the most universal law in the field of celestial mechanics, but for a long time, due to the complexity of galactic structure and multiplicity of celestial body motion, the law of universal gravitation has not been applied to a galaxy and the universe as a whole. But if we use the analytical methods of systems to treat a galaxy, or even whole universe, as a mass point, this problem will be easy to be solved.

\section{(1.1) What is the Galactic Center?}

The Galactic Center should be a large galaxy that it is at the same level of the galaxy structure as the Solar System and it is made up of a lot of the fixed stars. It is now widely regarded as a Black Hole, then why the center of the Solar System is not the Black Hole but the Sun? The Solar System is just a galaxy that it is at lower one level of the galaxy structures than the Milky Way.

Why is the Galactic Center a large galaxy at the same level as the Solar System? NASA astronomers have found dozens of large and very bright fixed stars near the center of the Milky Way galaxy using the Chandra X-ray space telescope (baike.baidu.com). According to the current popular theory, it is impossible to have any celestial bodies near black holes. Normally, any matter, including the fixed stars, will disappear without any trace when they fall into the gravitational field of the Black Hole. The celestial bodies near the Black Hole will gradually be swallowed up by the Black Hole, then why are these dozens of huge fixed stars not swallowed up by the Black Holes? The only reasonable explanation is that the imaginary Black Holes are not present. ${ }^{1}$ In addition, by using the radio telescopes, the astronomers have found a strong radio source at the Galactic Center, and call it Sagittarius $\mathrm{A}^{*}$ (coolcosmos.ipac.caltech.edu and baidu).
The center of the radio source is smaller, it is not larger than Jupiter's orbit around the Sun. Some people think that if the radius of the central core of the Milky Way is no more than 0.32616 light years, that is, no more than 0.3 light years, it means that it is likely to be the center of a large mass of dense body. That is to say that this dense massive celestial body, the Sagittarius $A^{*}$, is together with the dozens of fixed stars, to form the Galactic Center. It is a very similar structure as the Solar System, except that the Solar System consists of a fixed star and nine planets, but the Galactic Center is made up of dozens of fixed stars and a massive, dense body, a fixed star of a fixed galaxy, the Sagittarius A*. Sagittarius A* is equivalent to the Sun in the Solar System, and the dozens of huge fixed stars are equivalent to the planets in the Solar System. Therefore, the Solar System can be called a planetary galaxy, and the Galactic Center can be called a fixed galaxy.

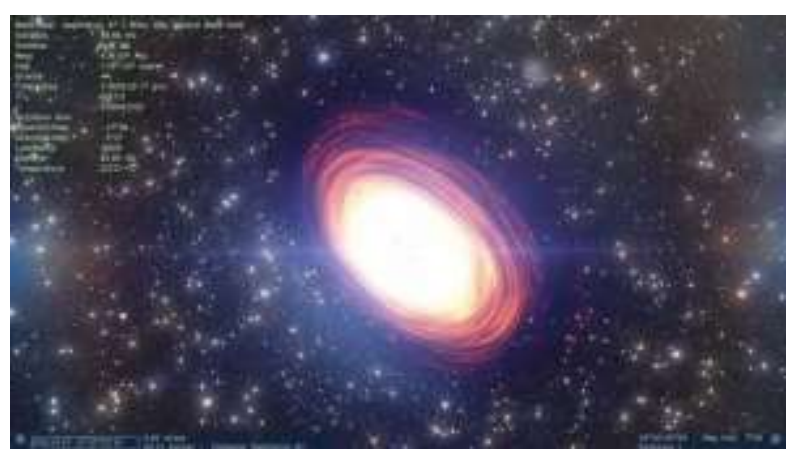

FIGURE 3: Sagittarius A*

\section{(1.2) Structure of galaxies}

Take the Solar System as an example, the Sun is positioned at the center of the system, and its position is relatively static, it is called a fixed star. Other celestial bodies are centered on the Sun and move around the Sun continuously, and they are called the planets. Therefore, in order to facilitate the study, we can call the central secondary galaxy a stellar galaxy, or a fixed galaxy, and call the peripheral secondary galaxies planetary systems, or planetary galaxies, which are moving around the fixed galaxy. A galaxy is made of a massive fixed galaxy, which locates the center of the system, and many peripheral planetary galaxies that moves around the fixed galaxy.

\section{(1.3) Systems analyses and the application of the law of universal gravitation}

Because of the distance between the same level galaxies is much farther than those between the secondary galaxies, such as, the distance between the Earth system and the Jupiter system is much farther than those between the Earth and the Moon or between the Jupiter and the satellite of Jupiter, so that when we analyze a galaxy, we can regard it as a mass point to study, and doesn't consider the secondary galaxies' specific state of force and motion.

1 According to the current observations, only a dense mass object, Sagittarius A*, has been observed, and no black hole has been observed. But at present, the academic circles do not believe what they observe, they believe that they are conjectured by a certain theory. 
For example, when we study the universal gravitation between the Earth system and the Jupiter system, we may take the two systems as two mass points, namely the Earth and the Moon as a particle, and the Jupiter and the Jupiter's moons as another particle, thus we can use the law of universal gravitation to calculate the force between the two systems, and do not consider secondary galaxies' specific state of force and motion, such as, the Earth and Jupiter, the Earth and the Jupiter's moon, the Moon and Jupiter, and the Moon and Jupiter's moon.

\section{(1.4) Relatively static and absolute motion}

For the Earth system, the Earth is in a relatively static at the center of the system; for the Jupiter system, Jupiter is in a relatively static at the center of the system, but for the Sun, the Earth and the Jupiter are moving around the Sun, they are in an absolute motion all. Similarly, for the Sun, in the Solar System, the Sun is in a relatively static, but the Sun is in the absolute motion in the Milky Way. The orbit of the Moon is fixed, and the orbit of the Earth is fixed, and the orbit of the Solar System and the orbit of the Milky Way can also be analogized, they are all fixed and in a relatively static state. That is to say, for a galaxy, the orbit of the secondary planetary galaxy is fixed and it is in a relatively static, but for the upper level galaxy of this galaxy, the secondary planetary galaxy's orbits are moving along with this galaxy's orbit, and it is in an absolute motion, for example, the orbit of the Moon is fixed for the Earth, but for the Sun, it is moving along the Earth's orbit. If we analyze the motions of a lower level galaxy in discontinuous Galaxy's levels, it is extremely easy to make the question becoming to the complicated and meaningless, such as, when we analyze the motion of the Earth system in the Milky Way Galaxy.

\section{(1.5) Resultant forces are zero and the orbit's fixed and unchanged}

Whether it is a celestial body or a galaxy, when it moves in its orbit, its external forces is always in equilibrium, that is, the resultant force is zero, if the resultant force is not zero, then its orbit can't be fixed and unchanged. The external forces include the universal gravitation between it and the same level galaxies, mainly means between the fixed galaxy and the planetary galaxies, and the centrifugal force which are generated when they move along an arc orbit. It can be said conversely that the balanced state of force is an important guarantee for a planet or a planetary galaxy to move regularly along a fixed orbit. Because the resultant force of a celestial body or a galaxy is always zero, therefore, it always moves regularly in its orbit.

\section{(1.6) Structural model of the Solar System in an ideal state and the cause of the elliptical orbit of a planet}

\subsubsection{Structural model of the Solar System in an} ideal state

Because we take a galaxy as a mass point to analyze its force and motion, therefore, it can be considered that the universal gravitation exists in between the same level of galaxies only. A galaxy will not exert any force on a secondary galaxy of another, and there are no forces between the secondary galaxies that are belonging to two same level galaxies respectively ${ }^{2}$. That is to say, when we analyze the force between the secondary galaxies of a galaxy, we should treat this galaxy as a closed system, and treat all the secondary galaxies of this galaxy as a number of particles to analyze. For example, when we analyze the force's state of the Solar System in the Milky Way, we should treat the Milky Way as a closed system, and regard the Solar System, the Galactic Center, and the Solar System's sibling galaxies as several particles, and only analyze the forces between the Solar System, the Galactic Center, and Solar System's sibling galaxies, and not take into consideration the force between the Sun and its planets, or the Sun and other galaxies of the Milky Way, or the force between the Solar System and the galaxies outside the Milky Way, and so on.

In an ideal model, we could take the Solar System as a closed system to analyze the forces between every planet, and only consider the impact internal of the Solar System, without considering outside of the system. In such a closed system, assuming that all the celestial bodies are a standard sphere, the mass of the Sun is the greatest, the other planets have the same mass, then their distribution in the system should be that the Sun is at the center of the system, the other planets are at the same distance from the Sun and symmetric distribution around the Sun. These planets make the uniform circular motion at the same speed and period along their own circular orbits having the same radius. Because it is only way to make sure the forces of the system are in an equilibrium state, and to ensure that the system can be a long-term stable existence! In this case, whole Solar System should be a standard sphere which is made of these planets and their orbits, that is, a standard large sphere.

\subsubsection{Cause of the elliptical orbit of a planet}

But in fact, the mass and shape of each planet in the Solar System are different, so the action of forces between them is constantly changing with their different spatial locations. In addition, some celestial bodies and galaxies outside the system also produce the action of forces with these planets, because the Solar System is in continuous motion, its external environment is constantly changing, and it is adjacent to different galaxies at different time, these adjacent galaxies vary greatly in shape, mass and distance. That is to say, the Solar System is not closed, it is always disturbed and influenced by the galaxies and celestial bodies outside the Solar System. Thus, the result of the combined effect of internal and external systems makes the orbits of these planets into elliptical. It can be said that the elliptical orbit of the planets in the solar system is the result of the balance of the internal and external forces of the solar system.

2 Because this galaxy is seen as a particle, its secondary galaxy is included in this particle. 
When the planet moves in its elliptical orbit, although the state of the forces is in changing constantly, but it can always reach a balance state of force. The balance of the force varies with the position of a planet in its orbit, and the speed of planet's motion is adjusted adaptively with the change of its equilibrium state, and can always ensure the planet moves regularly along the fixed elliptical orbit. At the different time in their orbits, the resultant force of the universal gravitation on the planets is always pointed toward the Sun, and it is with the direction of the centrifugal force generated that when it runs along the arched orbit is opposite and the magnitude is equal. The equilibrium state of force has certain periodicity along with the revolution of planets in the Solar System, and the period is the same as the revolution period of the planet. In other words, when the planets move in their orbits, although they are acted by the periodic forces from inside and outside the system, but they always are in a state of dynamic equilibrium of the forces. The dynamic equilibrium state of the forces is the guarantee of the regular movement of the planet along the fixed orbit. Although the shape of the Solar System, which is made of all planets and their orbits, is irregular, but it still has a spherical outline.

\subsubsection{Brake effects and the Virgin Mary and Saint - Baby phenomenon}

In the Solar System, every planet moves regularly in their respective elliptical orbit. When these planets are in aphelion orbit, they are far away from each other and the influence between them is negligible. But when they move on the perihelion orbits, the distance between them becomes small, and it will produce a significant effect on each other. Especially some massive planets, mainly the Jupiter, when it moves from the aphelion to perihelion orbit, the universal gravitation between it and the Earth increases, and the direction of the universal gravitation with the Earth's direction of spin is roughly opposite, so the Earth's spin be restrained correspondingly, and the Earth's spin slows down, and it produces a "braking effect" on the Earth. It has a subversive effect on the Earth's atmospheric circulation and ocean circulation, and we call it El Nino phenomenon.

When Jupiter moves from the perihelion to aphelion orbit, because its direction of motion changes, the direction of universal gravitation changes also, and it is roughly the same or similar with the Earth's spin direction, so it will weaken the binding force of extraterrestrial gravity on the spin of the Earth and causes the spin of the Earth is accelerated correspondingly, the result is it will form the antibrake effect, and we call it the La Nina phenomenon. If Jupiter goes from the perihelion to aphelion orbit, but the other massive planet moves from aphelion to perihelion orbit, then the action of the Earth's forces becomes more complicated, the La Nina phenomenon may weaken or disappear.

\section{(1.7) Mass distribution of a system and the central effect}

\subsubsection{Central effect phenomena}

In a galaxy, the fixed galaxy at the center of the system has the most mass, and other planetary galaxies are much, much less massive. For example, in the Solar System, the Sun is more massive than the planets, and in the Milky Way, the Galactic Center is much, much more massive than the Solar System and other planetary galaxies. When we analyze the effect of one galaxy on another, we consider mainly the effect of the central fixed galaxy (for example, the Galactic Center) on its peripheral planetary galaxies (the Solar System), which is making these planetary galaxy's (the Solar System) secondary planetary galaxy's (the Earth etc.) orbits to elliptical, and regardless of the effect between the planetary galaxies, because the mass of the planetary galaxy is are much less massive than the fixed galaxy, and the distances between two planetary galaxies is too far and too far, so the universal gravitation between them can be neglected. We call it the central effect phenomenon that because of the central fixed galaxy has the largest mass and so it also has the largest effect on other peripheral planetary galaxies. The fixed galaxy's (for example, the Galactic Center) central effect makes its peripheral planetary galaxies' (the Solar System) secondary planetary galaxy's (the Earth etc.) orbits to elliptical. It can be said the reason of the Moon's orbit is elliptical, and there is the perigee and apogee phenomena are mainly caused by the Sun, and the Earth's orbit is elliptical, and there is the perihelion and aphelion phenomena are mainly caused by the Galactic Center. The influence between the same level galaxies is mainly reflected in the central effect of the fixed galaxy. Interaction between planetary galaxies is just obvious when they are close to each other only, such as, the massive planet close to the Earth will have a certain impact. The interaction between galaxy and galaxy is mainly manifested as the elliptical orbit of secondary galaxies.

\subsubsection{The influence of galaxy's level on the central effect}

Generally speaking, the higher the level of a galaxy, the farther the distance between its peers. The less attractive one galaxy is to the planetary system of another (e.g., between the galactic center with the earth). That is to say, the interaction between galaxies of the same level gradually decreases with the increase of the level, the central effect of the central fixed galaxy decreases with the increase of the level. As a result, the transition of planetary system orbit from the ellipse of low-level galaxy to the circle of high-level Galaxy, at the highest level of the galaxy, the universe, the orbits of its subsystems are standard circles.

\subsubsection{Symmetry of mass distribution in high level galaxies and the idealization of the environment} 1.7.3.1 Convergence of the mass and the symmetry of mass distribution

The convergence of mass refers to the mass of each Secondary planetary galaxy tends to be equal in a galaxy as the level of the galaxy structure increasing, and the interaction between the same level galaxies tends to be the similar. 
The symmetry of the mass distribution refers to the planetary galaxies are symmetrically distributed around the central fixed galaxy. On the point of view of probability in mathematics and the point of view of the population balance of forces in physics that refers to the equilibrium of the interactions between all the celestial bodies in a system, for the planetary galaxies, the higher the levels of the galaxy structure is, the closer to equal their mass is, and the higher the degree of symmetry of mass distribution is.

In the Solar System, the masses of planets vary widely, and the mass distribution is less symmetrical, the asymmetry of mass distribution leads to the interaction of forces between planets and between planets and the Sun is constantly changing, the population balance of system forces must be coordinated with the external environment to achieve.

In Milky Way, its planetary galaxies' mass difference reduces, and the mass distribution's symmetry between the Solar System and its sibling planetary galaxies is more than the Solar System' planets, and the Solar System and its sibling planetary galaxies' interactions tend to be similar, but the population balance of system forces must be coordinated with the external environment to achieve yet.

For a Local Group of Galaxies that is a superior galaxy consisting of the Milky Way and its peers, the mass differences between the Milky Way and its peers reduce further, and the symmetry of the mass distribution increases further. For the population balance of system forces, the degree of coordination with the external environment decreases, but the degree of system self-regulation further improves. As the increasing gradual of the galaxy levels, the mass of sub-galaxies of a galaxy gradually tends to be equal, and the distribution of mass tends to be symmetrical, the population balance of system forces is gradually changing from coordination with the external environment to system's self-regulation ${ }^{3}$. For the universe, except central sub-system, its peripheral sub-systems are same absolutely in mass, shape, and the distance from central sub-system, they are symmetrically distributed around the central sub-system, the population balance of the forces in the universe is entirely the result of selfregulation.

\subsubsection{Idealizing of the external environment and} the zeroing of its influence

The idealization of the external environment refers to the external environment of a galaxy is more and more similar to the environment of the idealized Solar System model as the rises of the galaxy level ${ }^{4}$, that is, the interaction between the external environment and the internal structure in all directions gradually becomes similar, the influence of external environment on the internal structure of the system is gradually weakened, until the outer environment of the universe has no effect on its inner structure, it is exactly the same with the idealized Solar System model. Because of the mass each secondary planetary galaxy tends to be equal in a galaxy as the level of the galaxy structure increasing, and symmetrically distribute around the central fixed galaxy, so the interaction between the same level galaxies tends to be the similar. Besides, as the rises of the galaxy level, the distance between the high-level galaxies becomes farther and farther, the mass of them are getting larger and larger, and the symmetry is getting more and more, so the interaction is also gradually tend to similar between planetary galaxies and between planetary galaxies and fixed galaxy, result in the central effect of the fixed galaxy is getting weaker and weaker, it can be thought that a galaxy is affected in any direction and at any time by the same forces from other same level galaxies. It has the same result as being not affected by the external environment, or it is equivalent to being unaffected by the external environment.

So, the external environment of a galaxy is more and more similar to the environment of the idealized Solar System model. The zeroing of influence refers to the gradual weakening of the influence of external environment on the internal structure of galaxies until the external environment of the universe has no influence on its internal structure. Because of the interaction is tend to similar between planetary galaxies and between planetary galaxies and fixed galaxy ${ }^{5}$, the central effect of the fixed galaxy is getting weaker and weaker also as the galaxy's level rises, as a result, the planetary galaxy's orbit alters from the ellipses of the low level galaxies to the trendingcircular of the high level galaxies, and at the highest level of the galaxy, that is, the universe, the orbit of its sub-system is a standard circle, the central effect of the fixed galaxy is zeroing.

In the Solar System, for the Earth system, the influence of other eight planets on the Earth system is quite different. The planets with large mass, such as Jupiter, can cause El Nino and La Nina phenomena, but the planets with small mass can only cause El Nino and La Nina events. Another the center effect of the Sun makes the moon's orbit into elliptical, so the Earth is affected by the different forces of the Sun and other eight planets at different time, the environment of the Earth is very different from the environment of Solar System's idealized model.

In Milky Way, for the Solar System, the difference of influence between the Solar System and its sibling planetary galaxies reduces, not as much as Jupiter and other massive planets' influence on the Earth. The central effect of the Galactic Center is still very strong, the orbit of the Solar System's planets, such

\footnotetext{
${ }^{3}$ See analysis in 1.9.

4 Which the external environment of the idealized solar system model has no effect on its internal structure.

${ }^{5}$ It can be understood according to the universal gravitation, on the aspects of mass and distance.
} 
as Earth, is still elliptical ${ }^{6}$, but the environment of the Solar System is more ideal than the Earth.

For a Local Group of Galaxies, the Milky Way is in a more ideal environment than the Solar System. For the universe, its external environment is an empty space $^{7}$, so the external environment has no influence on the internal structure, which is exactly the same as the external environment of an idealized Solar System model.

The idealizing of the external environment and the zeroing of its influence is an inevitable result of convergence and symmetrical distribution of the mass, of course, it isn't happening overnight, but is a gradual process. Only at the highest level of the galaxy structure, namely universe, and the influence of the external environment on the internal structure will completely disappear.

\subsubsection{Universe is a huge standard sphere}

In general, the lower the levels of a galaxy is, the more asymmetrical the distribution of mass within it is, the greater the influence between the secondary galaxies is, the more irregular the external shape of the galaxy as a whole is, and its external shape does not tend to sphere, Such as the Solar System. Conversely, the higher the levels of galaxy are, the more uniform its internal mass distribution is, the interaction between secondary galaxies is tend to similarity, the external environment is more similar to the Solar System's idealized model, and the external shape is becoming more regular, and is more toward the sphere. At the highest level of galaxies, namely the universe, is a standard sphere, and only it is a standard sphere, it is just possible to maintain the force dynamic balance between subsystems and ensure the stable existence of the universe.

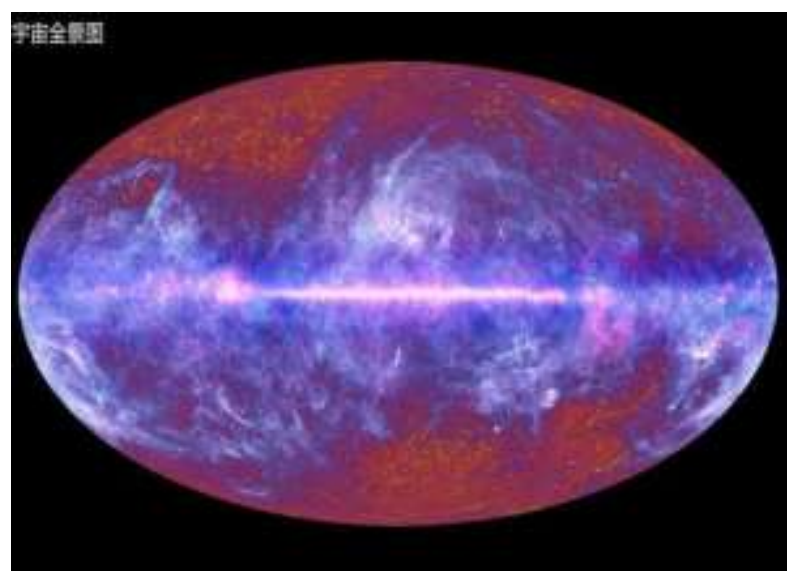

FIGURE 4: The universe

1.7.5 Factors affecting the external shape of a galaxy There are two main aspects:

The first is the impact within the system: the external shape of a galaxy is mainly determined by the mass of the fixed galaxy, the number of the planetary galaxies, and the mass and distribution of the planetary galaxies.
The second, the impact outside the system: the influence of the external environment on the galaxy's shape is mainly manifested on the central effect, that is, the same level fixed galaxy's central effect makes the secondary planetary galaxy's orbit of this galaxy to the ellipse. But the central effect decreases with the increase of galaxy's levels, therefore, the impact gradually returns to zero.

\section{(1.8) What is the outside of the universe?}

Suppose there are many akin-galaxy material systems in the external environment of a system called the "universe," according to the law of universal gravitation, these systems must interact with the "universe" system by the universal gravitation to form a larger system of matter, and the original so-called "universe" is really just a galaxy. In this way, the meaning is lost for the concept of "the universe." So, you can say the outer environment of the universe is an open and boundless space.

\section{(1.9) Could the universe exist in other shapes?}

Well, the universe, as a large system, and the galaxy, as a relatively small system, could they exist in other shapes? Such as, cubes, cuboids, or strips, ribbons, and planes? The answer is no! Because the premise is to maintain its stability for a system wants to survive for a long time. As you know, the only force to keep galaxies tightly connected and undivided is the universal gravitation, but universal gravitation alone can't keep the system stable for a long time, because it can only cause the celestial bodies to collide with each other and combine to a single body. Thus, it is necessary to require the constituent units of a system, namely the secondary galaxies or celestial bodies to move in a circular motion to generate correspondingly the centrifugal force to keep balance with the universal gravitation, such a state of equilibrium will necessarily require the system to be a spherical.

Then why is the universe a standard big ball? Because the outer environment of the universe is empty, and there is only the universal gravitation between sub-systems and the centrifugal force to keep the force's equilibrium of the universe, and is not affected by the external environment of the universe at all. Under these conditions, to maintain the force's population equilibrium and make the system stable existence for a long time, the system has to be a standard spheroid shape! Maintaining the population balance of a system's forces is the premise and foundation for the long-term and stable existence of a system. In such a system, its mass distribution must be uniform and symmetrical, that is, the sub-systems must be evenly and symmetrically distributed in space, the planetary sub-systems move around the central sub-system at the same radius, period, and speed to make a uniform circular motion, their mass must be equal, the shape must be similar, which is tends to the spherical but not standard.

6 The solar system is affected by its peers' galaxies is that its planets have elliptical orbits.

7 See analysis in 1.8 . 
Because the outer space of the universe is empty, if the sub-systems are difference in mass and shape, in the process of movement, the force between them must change and lose the balance, but it can't be regulated by the external environment in time, and result in the system can't maintain its equilibrium state of forces, and can't exist steadily for a long time. It is different from the lower level galaxies, although the low level galaxies are difference in mass and shape, but when the forces between them changes, it can be adjusted in time by the external environment, and it can keep the system in the balance, so it can survive for a long time and steadily. Hence, it can be said the population balance of forces of the lower level galaxies is the result of coordination with the external environment, but the population balance of the forces in the universe is entirely the result of selfregulation.

\section{(1.10) Reconsideration of the Solar System}

We know that, in the Solar System, except Uranus, almost all planets revolve around the vertical axis of the Sun in the same plane as the Earth, it seems that the shape of the Solar System is planar, what is going on here? There are two possible reasons for it: on the one hand, because of the Solar System is in a very low level of galaxy structure itself, hence, it has irregular external shape is reasonable. On the other hand, because planets on the same plane as the Earth are easy to be observed, while the planets of other spaces are not easy to be observed. If this view is correct, then there are far more than nine planets in the Solar System, just because they are not on the same plane with the Earth, far away from the Sun, and have a long period of revolution, so they are not easy to be observed in a short time. Only when planets are distributed in different planes, for example, the Uranus on the horizontal axis plane, and moving in different directions, such as, the Venus in clockwise direction, it is to be able to consistent with the idea of the population balance of forces.

\section{(1.11) "Ball in a ball" mode and multiple revolutions}

The universe, as a system of matter, exists in the shape of a standard large sphere, this sphere consists of many sub-systems, and these sub-systems take the center of the sphere as their symmetrical center, and this symmetrical center is also a sub-system, that is, the fixed galaxy sub-system, these sub-systems move in a uniform circular motion around the symmetrical center. Each sub-system is divided into many grandson systems, and these grandson systems take the center of the sub-system as their symmetrical center, and move regularly around this center in a nearly rounded elliptical orbit. The grandson system can also be divided to smaller micro-systems, so infinitely divided, until the lowest level of the universe structure, that is, the planets. It is just like a huge ball has many turning mediumsized balls in it, and there are many rotating small balls in each medium-sized ball, and these small balls are also filled with a number of rotating microspheres. Of course, for the external shape of a galaxy, the higher the levels of the galaxy structure are, the more tending to the spherical is, the lower the levels are, the more irregular it appears. In the whole universal system, the shape of a galaxy changes from the irregular shape of a lower level galaxy, but it has a spherical outline, to an almost a spherical shape of a high-level galaxy, up to the standard sphere of the universe.

\section{(1.12) Mathematics and the universe}

In the process of studying the universe's structure, the application of the limit thought of mathematics is very necessary. We can divide the galaxy structure of the universe into lots of levels, set to $\mathrm{N}$. The external shape of galaxies changes gradually from the irregular shape of the lower level galaxy to the standard large spheres of the universe; From lower level galaxy's difference on the shape and mass changes gradually to the sub-system's exactly same; From the lower level galaxies' difference on the speed, radius, and period changes gradually to the sub-system's exactly same; From asymmetry of lower level galaxy's mass distribution to entirely symmetry of sub-systems; From the low level galaxies' elliptical orbit changes gradually to a subsystems' circular orbit, and so on. For the galaxies' spatial structure, there is a continuous variation process from the irregular shape of low-level galaxies to the standard sphere of the universe.

\section{(1.13) The motion of the universe}

Because the outer environment of the universe is empty, so whole universal system doesn't engender any forces with the surrounding environment, and is in a state where the resultant force is zero, hence, the universe can only have two states of motion: One is absolutely static. In this case, whole universal system is an organic combination of absolute motion and absolute static, the absolute motion refers to the motion of its internal material system, and absolute static is for the whole universe. Another state of being is the whole universe to make the uniform rectilinear motion. In this state, the entire universe is an absolute system of motion. Then, which of these two states of being are more likely to exist? According to the dialectical materialism's movement is absolute and stillness is relative, it is more likely for the entire universal system to make the uniform rectilinear motion, and only in this way, it can just reflect the infinite and vast character of outer space fully.

\section{(1.14) Velocity of a planetary galaxy}

Suppose the mass of the fixed galaxy of a galaxy is $\mathrm{m} 1$, and a planetary galaxy is $\mathrm{m} 2$. M2 is making the uniform circular motion around $\mathrm{m} 1$.

From can be rolled out, it can be seen the velocity of a planetary galaxy or celestial body in uniform circular motion depends on the mass of the fixed galaxy and the distance between the planetary galaxy and the fixed galaxy, but has nothing to do with the mass of the moving body itself.

In summary of the part of mass:

Generally speaking, the universe is a large material system, which can be divided into many smaller subsystems, and these sub-systems can be divided into 
countless minor grandson systems, thus infinitely divided until the lowest level of the universe, that is, the planets. Regardless of the level, all galaxies exist in the form of a sphere consisting of numerous secondary galaxies and their orbits, and this sphere moves regularly in a similar circular elliptical orbit. In the changing process of the galaxy's structure from planet, a simple spherical object, to universe, a complex huge standard ball, the changes of internal structure as follows: on the one hand, the central effect of the fixed galaxy changes from strong to weak, and gradually returned to zero, manifested in the transition from elliptical to circular orbits of a planetary galaxies. On the other hand, the symmetry of mass distribution in the system is gradually enhanced, from the complete asymmetry of the lower level galaxies to the complete symmetry of the cosmic sub-system. The varieties of external environment are shown as an idealized changing process, which from lower level galaxy's internal and external coordination of the system to maintain the balance of forces to the self-regulation of the universe that the external environment does not produce the effect at all. The effect of the external environment on the structure of a galaxy is mainly manifested as the central effect of the fixed galaxy, which makes same level planetary galaxy's secondary planetary galaxy's orbit into elliptical. We may study a galaxy as a mass point, the universe is infinite size for the Earth we live on, but for its outer space, we can regard it as a very small, very small mass point.

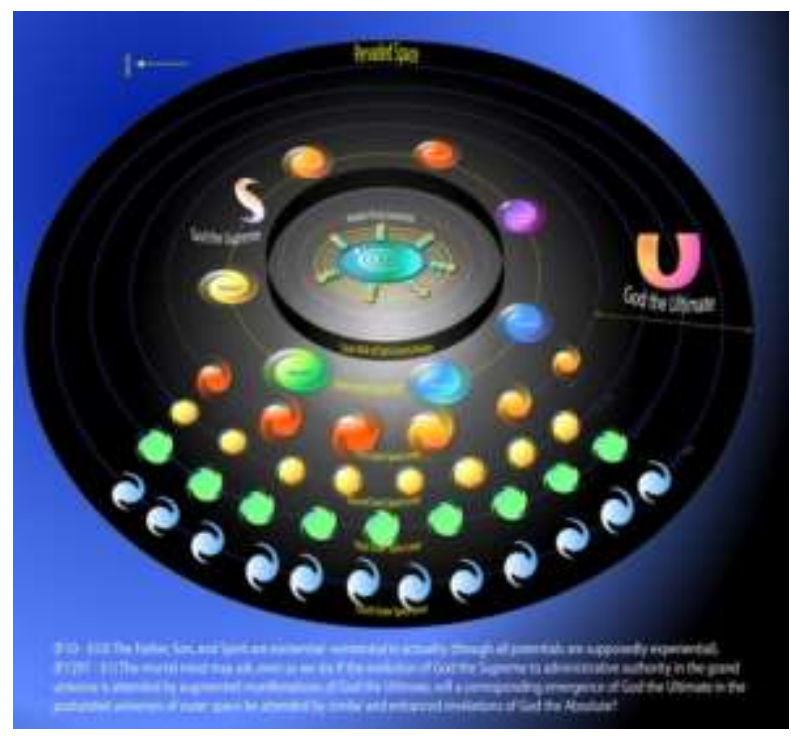

FIGURE 5: The structure of universe

\section{(2) Part of energy: the existence form of energy in the universe}

The universe is composed of many objective matters, and it is the unity body of space, matter, and energy. We know that the mass is distributing spherically symmetrical in the universe, then how does energy exist in the universe?

\section{(2.1) Gravitational potential energy of an object}

Airborne objects have the gravitational potential energy relative to the Earth's core. The Moon, which revolves around the Earth, should be essentially the same as the airborne object, and it is also located in the Earth's gravitational field, the universal gravitation also takes place between the Earth and the Moon, and it should have also the gravitational potential energy. But the motion between the Earth and the Moon is relative, for the Earth, the Moon moves around the Earth, but for the Moon, the Earth moves around the Moon, the moon has the "gravitational potential energy" in the Earth's gravitational field, but the Moon has also itself gravitational field, so the Earth should have also belonged to the Moon's gravitational field's "gravitational potential energy," for it is also in the Moon's gravitational field. The gravitational potential energies of the Moon relative to the Earth is numerically equal to that of the Earth relative to the Moon, and they are essentially the same thing, only because the angle of view of the problem, and produces two different statements, therefore, we might think that it is a gravitational potential energy shared by the Moon and Earth. The interaction way of forces between an airborne object and the Earth by the gravitational field, in essence, is identical with the interaction way of the Moon and the Earth, it's just the mass of airborne object is too small and form a gravitational field is extremely weak, so that people neglect its existence, but the gravitational potential energies generated by the gravitational field of an aerial object relative to the Earth is numerically equal to the gravitational potential energies generated by the Earth's gravitational field relative to the aerial object.

\section{(2.2) The gravitational field and the acting mode of force}

Therefore, we might as well give such a definition to the gravitational field: the field exists with the existence of mass, is the way of existence, operation, and transmission of energy, and the acting medium of the force. The direction of energy transmission and operation is consistent with the direction of the field strength. The strength of the field is positively related to the mass of the object, and inversely related to the distance from the object's centre of gravity, the direction of the field intensity is towards the object's centre of gravity. The direction of the field intensity between two adjacent objects is opposite, so the field intensities of these two fields can cancel out each other. The object's weightlessness state in space is rightly due to the counteraction of gravitational fields each other between celestial bodies. The operation and transmission of energy and the interaction of forces between objects are realized by the mutual cancellation of their gravitational fields ${ }^{8}$.

\section{(2.3) Conversion of the kinetic energy and the} potential energy of objects in free-fall

2.3.1 Conversion of the kinetic energy and the potential energy of objects in free-fall

Classical physics holds an airborne object is in freefall because of the gravity, the gravity is doing work

8 The way to do this is to do work on objects through universal gravitation. 
on it, the result of doing work is the gravitational potential energies is transformed into the kinetic energies of the object in free-fall. When an object falls in height, the gravity does positive work the gravitational potential energies is reduced, and the amount of gravitational potential energies reducing is equal to the doing work on it by gravity. When the height of the object increases, gravity does negative work, and the gravitational potential energies increases, the amount of gravitational potential energies increasing equals to the doing work on it by the object to overcome gravity. Then, when the object falls to the ground and remains motionless, because the ground is used as a reference plane, the gravitational potential energies of the object is zero, at the same time, the object is stationary and the kinetic energies of the object are also zero, that is to say, the object has neither gravitational potential energies nor kinetic energies of the free-fall. In this way, the energies of the object disappear without any reason, therefore, the view of classical physics has some cognitive limitations for this academic question.

\subsection{2 in a resting state, the kinetic energy of an object on the ground}

An airborne object's free-fall process is essentially a process that the Earth's gravitational field counteracts the object's gravitational field, and exerts force between them, and transmit finally the energies of the objects to the Earth, the energies transfer is achieved by the gravity to do work on objects. Then, what form energy of the object can be transmitted to the Earth? Is it the gravitational potential energy of the object, or some other form of energy? As we all know, the Earth spins constantly around the Earth's core for 24 hours, in the course of the Earth's spin, whether an airborne object, assuming it is at rest, or an object on the ground move all in a uniform circle around the Earth's core, so they has the kinetic energy of the uniform circular motion. The airborne object is farther from the Earth's core than the ground's one, its linear velocity is greater than the ground, so it has more circular motion's kinetic energies than the ground. When it falls from the air to the ground, where does the difference of the kinetic energies go? We can only think it was passed to the Earth, as for the process of doing work on the object by gravity can only be used as a way of transformation and transmission of the energies, but it can't eradicate the existence of energies fundamentally. In that way, what form of energy does the difference of kinetic energies exists after it is propagated to the Earth?

\subsubsection{Law of universal gravitation and the gravitational potential energy}

The distance between the airborne object and the Earth's core becomes smaller in the course of freefall, according to the law of universal gravitation, the universal gravitation between the object and the Earth becomes larger, accordingly, the field strength of the gravitational field of the Earth should increase with the shortening of the distance between two, and the gravitational potential energies between two should also increase rather than decrease. Therefore, the traditional idea in the free-fall process of an object, the gravitational potential energies are transformed into this object's kinetic energies in free-fall, the gravitational potential energies of the object decreases, and the kinetic energies of free-fall increases is a wrong understanding for the energy conservation law. It's essentially the object in the free-fall, the distance between the object and the Earth's core becomes shorter, its linear velocity of the uniform circular motion, which is arising from the spin of the Earth, decreases, and its kinetic energies of uniform circular motion decreases accordingly. As for which part of the object is losing energy in the circular motion, one part of it changes into the kinetic energies of the object in free-fall, and the other is converted into gravitational potential energies between the object and the Earth. In this way, the object transfers the kinetic energies difference to the Earth by increasing the gravitational potential energies between it and the Earth's core. When the object stops at the Earth's surface, its kinetic energy of free-fall is also converted into gravitational potential energies between it and the Earth's core and eventually into the internal energies of the Earth.

The essence of universal gravitation action between two objects is a process of the operation and transmission of energy between them by their gravitational field as a medium. The operating and transitive way of energy is to do work on objects by universal gravitation, the result of doing work is the kinetic energies are converted to the potential energies, and make two objects have a stronger mutual attraction and further combine into one's energy trend. When two objects combine into one, the gravitational potential energies of them are also combined into one, as a result, energies fusion occurs, and the potential energies are converted into the internal energies of new object, and put the newly combined body in a metastable state caused by energy.

The essence of force action lies in the operation of the gravitational field to energy. The objective existence of the energy is one of the first, only when energy exists objectively, then it is possible to produce the force's interaction by the operation of the gravitational field to energy. Rather than to occur firstly action of force, and then causes the operation of gravitational field and result in the production of energy final ${ }^{9}$. Operating way of the gravitational field is to cancel each other the gravitational field between two adjacent objects.

\section{(2.4) Energy transformations of planets during motion}

2.4.1 The conversion of kinetic energy and potential energy of planets in motion

${ }^{9}$ The classical physics holds that the gravitational potential energy of an object is generated by the action of gravity. 
In the Earth system, the Moon is centered on the Earth for inertial motion. If there is no Sun, the Moon moves at a uniform speed around the Earth in a circular orbit. However, due to the central effect of the Sun, the Moon's original circular orbit is drawn into the ellipse. On this elliptical orbit, when the Moon moves from apogee to perigee orbit, the distance between the Moon and the Earth becomes short, and the universal gravitation between the two is enhanced. This universal gravitation not only points to the Earth, becomes the centripetal force of the Moon for circular motion, and because the Moon's orbit is elliptical and eccentric, the universal gravitation also produces a tangential component of the direction of Moon's motion and causes the Moon to be accelerated. The speed of the Moon gradually increases, and when it gets to the perigee orbit, its speed approaches the fastest. When the Moon moves from perigee to apogee orbits, because of the direction of motion's changing, so the speed of Moon's changing is opposite, the speed of Moon gradually becomes slower, and when it reaches apogee orbit, the Moon's speed is the slowest.

When the Moon moves from apogee to perigee orbit, the distance between the Moon and the Earth becomes small, and the gravitational potential energies between two increases, at the same time, the speed of the Moon's movement gradually increases, its kinetic energies also increases, then where does increasing this part of the energies come from? It can to be analyzed on the point of view of central effect of the Sun, because the distance between the Moon and the Sun changes, the gravitational potential energies between them changes also accordingly, when the distance between two increases, the gravitational potential energies between them decreases, the reduced gravitational potential energies are transformed into the increased gravitational potential energies between the Moon and the Earth and the increased Moon's kinetic energies. When the Moon moves from perigee to apogee orbit, the opposite is true, the gravitational potential energies between the Earth and the Moon will reduce due to the distance between them becomes big, and the kinetic energies of the Moon reduces also due to its speed becomes slow. The reduced gravitational potential energies and kinetic energies will be transformed into increased gravitational potential energies between the Moon and the Sun. This pattern of variation also may be applied to planets in the Solar System.

In the Solar System, when two planets move from aphelion to perihelion orbit, the distance between them is shortened, the gravitational potential energies between them is enhanced, and the enhanced gravitational potential energies are transformed from the kinetic energies of their inertial motion, which is reflected in the adjustment of the planet's speed to adapt the running environment, and the planet's speed becomes slower. Will this conversion of kinetic energies and gravitational potential energies lead to the collision of two planets and merge into one? The answer is No. It is due to excessive kinetic energy of planet's inertial motion on its orbit and reverse attraction of other celestial bodies inside and outside the system, which makes it is very unlikely the two will collide and merge into a single body.

\subsubsection{The effect of energy's transformation on the Earth} 2.4.2.1 Impact on Meteorology

Due to the distance between two planets becomes short, their gravitational potential energies will increase, and the enhanced gravitational potential energies come from the inertial kinetic energies of planets, and is finally converted into the internal energy, and make the total amount of internal energies planets increase. The increase of the total internal energies will have a major impact on the Earth's meteorology, atmospheric circulation, and ocean circulation. Such as El Nino and La Nina phenomenon, climate warming, frequent extreme weather, and tidal changes etc. These impacts show a certain degree of directionality and periodicity, the directivity is mainly shown in the relative direction of the planet and the Earth, the periodicity is related to cycles of this planet and the Earth around the Sun.

\subsubsection{The effect on the force}

One is the impact on the Earth's crust movement, in essence, it should belong to the category of the exogenic force. The second is to change the direction and speed of the Earth, of course, the change is small and instantaneous, and the population balance of force can be achieved by the environment's adjusting in time.

\section{(2.5) Formation mechanisms of solar energy}

\subsubsection{Formation mechanisms of solar energy}

In the Solar System, the planets, including the Earth, move regularly in their respective elliptical orbits around the Sun, each planet depends on its own mass to form its own gravitational field, but because the resultant force of universal gravitation of every planet point all to the Sun, so it can be inferred the direction of comprehensive field intensity of all planets' gravitational field also points to the Sun. Therefore, it can be considered the gravitational potential energy only occurs between the Sun and the planets, there is not the gravitational potential energy between planets (for ease of study). Due to the Sun form the gravitational potential energies with all planets, so it has a high potential energies value, but the Sun is relatively stationary in the Solar System, there's no centrifugal force to do work on it ${ }^{10}$ , these potential energies can't be converted into kinetic energies, namely, they can't exist in the state of kinetic energy, the only state is it can be transformed is the internal energy. When these potential energies are transformed into internal energies, the Sun would have a very high temperature and propagate energy outward in the form of light. Jupiter's thermal energy, like the Sun's, has the same form mechanism.

${ }^{10}$ See the analysis of 2.5.3.2. 


\subsubsection{The total internal energy of the Sun}

In an ideal state, that is, without the influence of external environment of the Solar System, the potential energies of the Sun are equal to the sum of the potential energies of all planets, therefore, its total amount of internal energies is equal to the sum of potential energies of all planets.

\subsubsection{Mutual transformations of kinetic energy and potential energy in the Solar System}

\subsubsection{Kinetic energy is transformed into potential energy}

Suppose in the absence of centrifugal force, the planets have to move towards the Sun under the action of universal gravitation, we can think the movement of planets to the Sun is a process of doing work on the planet by the universal gravitation, the result of doing work is to transform the inertial kinetic energies of the planet into the gravitational potential energies between planet and Sun, and make the gravitational potential energies between the two increasing, finally, the planet is combined with the Sun, and its potential energies are transformed into internal energy of the sun, Sun's internal energies increases.

\subsubsection{Potential energy is transformed into kinetic} energy

On the contrary, if there is no the universal gravitation, for a planet in an arc orbit, it will move inevitably in the direction of leaving the Sun because the centrifugal force, it can also be considered the centrifugal force is doing work on the planet, it make the gravitational potential energy between them is transformed into planet's inertial kinetic energy at the tangent direction, the gravitational potential energies of them reduces, the reduced gravitational potential energies is converted into planet's inertial kinetic energies, and Sun's internal energies decreases.

2.5.3.3 Energy conservation in the conversion of internal energies, potential energies, and kinetic energies

We know when a planet moves on its orbit, it is always in a state of dynamic equilibrium of forces, so the gravitational potential energies between it and the Sun is equal to planet's inertial kinetic energies, hence, it we can infer the sum of the potential energies of all planets is equal to the sum of the kinetic energies of all planets. But the sum of the potential energies of all planets is equal to the potential energies of the Sun, so the potential energies of the Sun is equal to the sum of the kinetic energies of all planets. Because the total potential energies of the Sun are equal to its total internal energies, therefore, under ideal conditions, the total internal energies of the Sun is equal to the sum of the kinetic energies of all planets. In the course of the inertial motion of a planet in its orbit, as its spatial position is constantly changing, its kinetic energies and potential energies are constantly transforming with each other. But for the Solar
System, if the environmental impact is not considered, its total amount of energies is in conservation.

\subsubsection{Sun: activity}

2.5.4.1 Reasons outside the system

In fact, the planets not only interact with the Sun to form the gravitational potential energy, but also interact with the galaxies outside the Solar System to form the gravitational potential energy. It is mainly reflected in the central effect of the Galactic Center that it makes the orbit of the planets of the Solar System into elliptical. For the Sun, because of between Solar System's planets and the Galactic Center form the gravitational potential energy, so it increases the gravitational potential energies of the Sun in that direction, the Sun's internal energies in this direction also increase, it accumulates the energies for the Sun to enter the active period, and forms the external causes of the Sunspots and solar flares in the solar active region. The impacts are continuous and change periodically as the Solar System's revolution. External causes can only be in a position secondary contradiction.

\subsubsection{Reasons within the system}

Because the planets are moving in their respective orbits constantly, so at different time, their positions in the Solar System are different, the gravitational potential energies formed between them and the Sun also changes constantly, and Sun's total internal energies also changes, thus forms the solarmaximum and solarminimum of solar activity, and is the intrinsic causes of Sunspots and solar flares in the solar active region. At the solarmaximum, the planet's inertial kinetic energies are transformed into the potential energies between them and the Sun, and the Sun's internal energies increases. At the solarminimum, the potential energies are transformed into the planet's inertial kinetic energies, and the Sun's internal energies decreases. This change has a certain degree of directionality and periodicity ${ }^{11}$.

2.5.4.3 Jupiter's revolution and the Sunspots and solar flares

Sunspot's cycle is based on the Jupiter's period of revolution (Wu Yue-ming, 2016). When Jupiter moves from perihelion to aphelion orbit, Jupiter is getting further and further away from the Sun, the gravitational potential energies between the Sun and Jupiter reduces, the gravitational potential energies are converted into the inertial kinetic energies of Jupiter's, the internal energies of the Sun at that direction decreases, Solar activities shift from solarmaximum to solarminimum, the Sunspots and solar flares outbursts gradually fade.

When Jupiter moves from aphelion to the perihelion orbit, the distance between Jupiter and the Sun is getting closer and closer, the gravitational potential energies between them increases, the internal energies of the Sun at this direction increases also, the solar activities become intense, solar activities

\footnotetext{
11 on the point of view of the law of conservation of energy, the energy conversion and transmission between planets and the sun are related to the central effect of the Galactic center. See the analysis in 2.4.1.
} 
change from solarminimum to solarmaximum, and the Sunspots and solar flares are strengthened.

When Jupiter intersects with other planet at the nearby of perihelion orbit, the result of three celestial bodies close to each other is the gravitational potential energies between them increases large, and internal energies of the Sun increases large, make the solar activities becoming intense, and the eruption of Sunspots and solar flares intensifies.

When Jupiter intersects with other planets in a straight line at the perihelion orbit to pin down the Sun, the distance between three are nearer, the gravitational potential energies between them are more much, it make the Sun has more internal energies, and solar activities are more intense, the burst of Sunspots and solar flares are stronger. Increased potential energies are transformed from the inertial kinetic energies of Jupiter and the other planet. Other huge planets have the same effect on solar activities, and the effect depends on planet 's mass. The combined effects of multiple planets may result in the loss of regularity of solar activities, but compared to Jupiter, these planet 's mass are much smaller, and the impact is not as obvious as Jupiter.

When Jupiter moves from aphelion to perihelion orbit, the distance between Jupiter and Earth, between Jupiter and the Sun becomes closer all, and it has different effects on the Earth and the Sun. For the Earth, it causes the El Nino and La Nina phenomena. For the Sun, it causes the solar activity.

\subsubsection{The influence of the solar wind on the meteorology of the Earth}

When the Sun's internal energies increase at a certain direction, due to energy's accumulation and increase will cause its outer atmosphere to active violently and radiate energies outward, the radiation of energies travels outward in the form of the solar wind, and produce a certain impact on the Solar System's planets. For the Earth's meteorology, the solar wind blows towards the Earth cause mainly high temperature and drought in some areas, while other areas are in waterlogging. Reason is perhaps the solar wind's high-energies particles collide with atmospheric molecules and further combine into a number of bigger molecules, and newly formed molecules can become ice nucleations of the water vapors condensation. But because of the impact of high-energies particle flow, water vapors can't condense into ice with these ice nucleations as the core, the precipitation process can't be formed, makes these areas in Sunny, high temperature, and high humidity meteorological conditions. Moreover, due to high surface temperature causes water vapors continuously evaporate and rise, thereby forms a high pressure at the upper air and a pressure gradient with surrounding areas. Under the action of pressure gradient force, water vapors and the newly forming ice nucleations move together along the pressure gradient, and get to the surrounding areas. The direction of its movement is affected by the topography, landforms, and atmospheric circulation. When these water vapors and ice nucleations come to the sky over another area, because of the temperature decreases, the water vapor reaches a saturated state, and there is no interference of the solar wind's high-energies particles, so these water vapors can be combined into ice, and causes a rainfall process. Therefore, when the solar wind blows to the ground, it always can cause the high temperature and drought in one area and the waterlogging in another.

\subsubsection{The impact of the Solar System's revolution on the Earth}

2.5.6.1 Impact on meteorology

In the Milky Way, the Solar System revolves around the Galactic Center along an elliptical orbit, when it moves on the pericenter orbit, the distance is relatively close between the Solar System and Galactic Center, the gravitational potential energies of them increases, the enhanced gravitational potential energies are converted further to the internal energies, it makes whole Solar System's temperature rises. For the Earth, it should be in a warm, humid climate, namely a warm-wet period ${ }^{12}$.

When the Solar System moves on the apocenter orbit, on the contrary, it should be in an ice age. During the warm-wet period, the Earth's climate is like the summer all year round, glaciers melt, sea levels rise, deserts disappear, wetlands increase.... ${ }^{13}$

During an ice age, the Earth's surface temperature drops, the water of surface freezes in the middle and high latitude areas, the total amount of water vapors of the atmosphere decreases, and the air is cold and dry, four seasons in a year. When the Solar System moves on the apocenter orbit, if it is adjacent to other sibling's galaxy, the gravitational potential energy between the Solar System and its siblings increases, the temperature of the solar system also rises, and the earth is in the interglacial period. When the Solar System moves on a transition orbit of between the apocenter and pericenter, the distance between the Solar System and the Galactic Center is also relatively close, and the Earth's climate is still warmer and moister, but it is in the interim period between the ice age and the warm-wet period ${ }^{14}$.

\subsubsection{Impact on geomechanics of the Earth}

When the Solar System runs on the pericenter orbit, the distance between the Solar System and Galactic Center is nearer, the universal gravitation between two is enhanced. But as the Solar System moves on an arc orbit that has a larger radian, so the direction of motion is constantly changing, the Solar System's force state is prone to be in a brief imbalance state,

\footnotetext{
12 Which is precisely the dinosaur era.

13 From the extinction of dinosaurs to now, the earth has been in the ice age.

14 The whole Cretaceous (about 70 million years) is in this transitional period (See analysis below). In the late Cretaceous, the earth began to enter the ice age.
} 
and there are an alternating changes phenomenon of "the temporary imbalance state" and "the rebalance state after adjustment inside and outside the system" along with its spatial position's changing. It should produce a significant impact on the geomechanics of the Earth and other planets. The crustal movement becomes intense, and the mountain-building activities occur on the Earth's surface, and the natural disasters, such as, earthquakes, volcanic eruptions, tsunami, landslides, and mudslides, etc. are frequent. In "the temporary imbalance state," because the solar system is in an imbalance of forces, the crustal movement of its inner celestial bodies is intense, the Earth is in an active period of the orogeny. And in "the rebalance state after adjustment inside and outside the system," because the solar system is in a balance of forces, the crustal movement of its inner celestial bodies is abated, the Earth is in a dormant period of the orogeny. When the Solar System moves on apocenter orbit, the influence of the Galactic Center on the internal structure of the Solar System can to be negligible because of the distance from the Galactic Center is far away.

The most recent mountain-building activities on Earth occurred in the Cretaceous Period 100 million years ago ${ }^{15}$ (Reidy, Michael S, 2017), on the climate point of view, it belonged to warm-wet period at the dinosaur life era, and the Solar System should move on a transition phase orbits of the pericenter and apocenter. It can be inferred from the evolutionary process of the angiosperm (Zhang Mingzhen, etc., 2018), since the " pericarp " of angiosperms is a protective tissue that the plants have evolved to adapt to a bad environment, and angiosperms appeared in early Cretaceous, and had a substantial increase in medium term, and it obtains a dominant position in terrestrial plants at the late period, shows the cretaceous period was a transition period of the Earth's climate from warm-wet period to ice age, the Solar System's position should also be in a transition stage from the pericenter to apocenter ${ }^{16}$.

\section{(2.6) Formation mechanisms of the thermal energy of Earth's core}

\subsubsection{Formation of the thermal energy of Earth's core} As we all know, the Earth's crust can be divided into six plates: the eurasian plate, the african plate, the american plate, and the pacific plate, the Indian ocean plate, and the antarctic plate. Between every plate and Earth's core should also form the gravitational potential energy, in the absence of normal force and adhesive force's effect, the sum of gravitational potentials energies of all plates should also be equal to the gravitational potential energies of Earth's core. As the Earth's spin, these plates move at a uniform circle motion around the Earth's core, but the Earth's core is in a relatively static state, there's no centrifugal force to do work on it, therefore, so much gravitational potential energies is not likely to be converted into the kinetic energies, and exists in the form of the kinetic energy, in the end, it can only be converted into internal energies, and at the thermal energy of the Earth's core to exist. Like six major plates, the gravitational potential energy between the Moon and the Earth is likewise a source of the energy of Earth's core and a source of heat for surface temperatures.

\subsubsection{Genesis of volcanoes and crustal movement}

Since Earth's core has a high value of the thermal energies, there must be a temperature difference between it and the six plates. The temperature differences must cause thermal energies to travel from a high temperature part to the low. However, because the six plates are not exactly same in geological structure, the thermal conductivity of soil in some areas is high, and some areas is low. Where the thermal conductivity is high, the thermal energy travels fast and much, and the influx of thermal energy will cause the Earth crust's rocks to be melted into liquid, that is, magma, which is a highly unstable state, something like a metastable state, but it is a high-energy state. In this state, if Earth crust moves, it is very easy to cause magma to erupt outwards, that is, the so-called volcanic eruption, and it is why volcanic activities are often associated with Earthquake. The reason why Earth crust is divided into six plates is just because the thermal conductivity of plate's junction is high, the influx of large amounts of thermal energy causes Earth crust to be melted and become liquid and then the Earth shell was torn. After Earth crust is split into six plates, if other planets, even the Moon, approach the Earth, under the perturbation of its universal gravitation, the crustal movements are extremely easy to occur. It is so faint and is hard to be felt at the center of plate, however, at the edge of the plate. The motion is easy to be detected because of the motion state's difference of plates, and this is why the Earthquakes and volcanic eruptions occur usually at the junction of the plates.

\section{(2.7) Existence of Energy in the universe}

To sum up, in the entire universe system, the kinetic energy and potential energy are the existing rudimentary forms of energy, and the internal energy is a complementary form of energy under the condition of relative rest. The essence of force action lies in an operation of the gravitational field to energy ${ }^{17}$, which is achieved by force to do work on an object. The energy's objective existence is primary, only when energy exists objectively, then it is possible to appear force's interaction by the operation of the field.

\footnotetext{
15 At previous period, the orogeny is in an active period. In the next 100 million years, the solar system is far away from the Galactic center due to the solar system on the apocenter orbit, the influence of the Galactic Center on the internal structure of the Solar System can to be negligible, the Earth is in a dormant period of the orogeny.

16 The temperature of the earth changes from hot to cold, which means that the internal energy changes from more to less, the gravitational potential energy between the solar system and the Galactic Center also decreases, and the distance between the two gradually increases.

17 The operation mode of energy is realized by universal gravitation to do work on the object.
} 
Therefore, the statement in classical physics that the gravitational potential energy is possessed by an object because of gravity's action, is a distortion of the relationship between force, gravitational field, and energy. There is firstly the objective existence of energy, then it is a possibility to produce an action of force, instead of there is firstly the action of force and then produce the energy later. At any levels of the galaxy, there is a numerical equivalence relationship between the potential energies of fixed galaxy locating in system's center and the sum of the kinetic energies of all planetary galaxies. Because the outer environment of the universe is empty, therefore, it should not form gravitational potential energy with the external environment and produce transfer and exchange of the energy, so the universe's total amount of energy is constant. The exchange of energies between same level galaxies is balanced, so even though the Sun is constantly sending energies out in the form of light, but the celestial bodies outside the Solar System either transmit energies to it in the form of gravitational fields or in the form of light to maintain the balance of energies transfer, so that the Sun has an inexhaustible source of energies.

\section{(2.8) Energy levels division of galaxies}

\subsubsection{Kinetic energy levels division of galaxies}

For galaxy's structure, there are $\mathrm{N}$ levels from Moon

to the universe, the Moon's mass is $\mathrm{m}^{1}$, the Earth's

mass is $m^{2}$, excluding the Moon, the Sun's mass is $m$

3 excluding the Earth etc....The velocity of the

universe's uniform rectilinear motion is $\mathrm{v}^{1}$, the velocity of sub-system revolving around universe's

center is $\mathrm{v}^{2}$, the velocity of grandson system of a sub-

system is $\mathrm{v}^{3} \ldots$ The revolution velocity of the Solar

System is $\mathrm{v}^{n-2}$, the Earth's revolution velocity is $\mathrm{v}{ }^{n-1}$

, the velocity of the Moon is $\mathrm{v}^{n}$. then for the uniform rectilinear motion of the universe, we can obtain the kinetic energy of each planet is that the kinetic energies of the Moon is $1 / 2 \mathrm{~m}^{1} \mathrm{v}^{2}{ }_{2}^{2}$, the kinetic energies of the Earth is $1 / 2 \mathrm{~m}^{2} \mathrm{v} 1$, the kinetic energies of the Sun is $1 / 2 \mathrm{~m}^{3} \mathrm{v}^{2}$. And for the revolution of the sub-system, the kinetic energies of the Moon is $1 / 2 \mathrm{~m}^{1}{ }^{2} \mathrm{v}^{2}$, the kinetic energies of the Earth is $1 / 2 \mathrm{~m}^{2} \mathrm{v}^{2}$, the kinetic energies of the Sun is $1 / 2 \mathrm{~m}^{3} \mathrm{v}^{2}$, etc. their total kinetic energies is

the Moon : $1 / 2 \mathrm{~m}^{1} \mathrm{v}^{1}+1 / 2 \mathrm{~m}^{1} \mathrm{v}^{2}+1 / 2 \mathrm{~m}^{1} \mathrm{v}^{2}$ $+\ldots . .+1 / 2 \mathrm{~m}^{1} \mathrm{v}^{2}$

the Earth : $1 / 2 \mathrm{~m}^{2} \mathrm{v}^{2}+1 / 2 \mathrm{~m}^{2} \mathrm{v}^{2}+1 / 2 \mathrm{~m}^{2} \mathrm{v}^{2}$ $+\ldots . .+1 / 2 \mathrm{~m}^{2} \mathrm{v}^{2}-1$

the Sun : $1 / 2 \mathrm{~m}^{3} \mathrm{v}^{2}+1 / 2 \mathrm{~m}^{3} \mathrm{v}^{2}+1 / 2 \mathrm{~m}^{3} \mathrm{v}^{2}$ $+\ldots \ldots+1 / 2 \mathrm{~m}^{3} \mathrm{v}^{2}$

If $M$ is used to represent an abstract conceptual mass of a planet, then the total kinetic energies of this planet is as follows:

$1 / 2 \mathrm{Mv}^{1}+1 / 2 \mathrm{Mv}^{2}+1 / 2 \mathrm{Mv}^{3}+\ldots \ldots+1 / 2 \mathrm{M} \mathrm{v}^{n}$

Then its energy levels of the kinetic energy are respectively:

First level $: 1 / 2 \mathrm{Mv}^{2}$

Second level : $1 / 2 \mathrm{Mv}^{2}+1 / 2 \mathrm{Mv}^{2}$

Third level $: 1 / 2 \mathrm{Mv}^{2}+1 / 2 \mathrm{Mv}^{2}+1 / 2 \mathrm{Mv}^{3}$

The $\mathrm{K}$ level : $1 / 2 \mathrm{Mv}^{1}+1 / 2 \mathrm{Mv}^{2} \stackrel{2}{2}^{2}+1 / 2 \mathrm{Mv}^{3}$

$+\ldots . .+1 / 2 \mathrm{M} \mathrm{v}^{2}$

The $\mathrm{N}$ level : $1 / 2 \mathrm{Mv} \stackrel{2}{1}^{2}+1 / 2 \mathrm{Mv}^{2}+1 / 2 \mathrm{Mv}^{3}$ $+\ldots . .+1 / 2 \mathrm{M} \mathrm{v}^{n}$

If the concept of the abstract mass is extended to galaxies, this method of dividing kinetic energy levels can be applied to any level galaxies.

2.8.2 Chaotic states of the asteroid and the activation Generally speaking, the lower the levels of galaxy structure is, the higher the kinetic energy levels is, and the most likely to be activated by the energy of external environment is, and to be in a chaos and deviate from original orbit, and collide with other celestial bodies or join other galaxies to find a new balance of force and energy. For instance, the universe at the highest levels of the galaxy structure has the lowest kinetic energy levels, and it has only one first energy levels $1 / 2 \mathrm{Mv}$ and it is impossible to be activated. But at the lowest levels of the galaxy structure, namely the planets, its kinetic energy levels are the highest, it is most likely to be activated by the energy of the external environment. However, due to some factors, such as excessive mass, the total energy conservation of the system, and balance in transmission, etc. for a big planet, it's unlikely to be activated. But for an asteroid, there is some possibility, for example, the large mass planet's perturbation, transient unbalance of forces in the system, and high energy plasma flow' erupt of the system center, etc. they can activate the asteroid and make it in a chaos. In fact, when a rocket is at rest on the Earth's surface, we may think it is traveling in a uniform circular motion around Earth's core, and the process we give it enough energy to send it into space can be seen as an activation process. In the vast void of space, comets are also probably celestial bodies to be activated.

\section{(2.9) The interface existence of life}

For any levels galaxy, the central fixed galaxy has the most energy and the highest temperature, so the possibility is the smallest there is life phenomenon, it should be said there is no possibility of life, we call the life can only appears on the surface of the system's phenomenon the interface existence of life. For the universe, there is impossibly to occur life in 
the central sub-system; For a sub-system, the life phenomenon can't occurs in the central fixed galaxy; In the Milky Way, there is no possibility of life in the Galactic Center; For the Solar System, there can be no life on the Sun; But for the Earth, it is a big exception, because the Earth has only one Moon around it movement, so the gravitational potential energy between them will not affect the formation of life on Earth, and that it should be a temperature protected umbrella of the life on Earth.

\section{In a summary of part of energy:}

Kinetic energy and potential energy are the existing basic forms of energy in the universe, and the internal energy is a complementary existing form of energy under relativity rest condition. The essence of force action lies in the operation of the gravitational field to energy, without the objective existence of energy, there would be no operation of the gravitational field and then appears the interaction of forces. The operation mode of energy is realized by universal gravitation to do work on the object. Force between objects is achieved by the two adjacent object's gravitational field to cancel each other. Because of the multiple motion of the universe, the kinetic energy in the universe exists on the division of energy levels, the higher the levels of the galaxy structure are, the lower the kinetic energy levels are. On the contrary, the lower the levels of a galaxy structure are, the higher its kinetic energy levels are, and it is easier to be activated and, in a chaos,, and deviate from the original orbit and join the new system to seek a new balance of the force and energy. For any level of galaxy, the fixed galaxy at its center always has the most internal energies, so the life can only exist in the surface of a galaxy, namely the planetary galaxies.

\section{CONCLUSIONS}

The universe is a large material system, which can be divided into many small sub-systems, and the subsystems can be divided into many minor grandson systems, thus infinitely divided until the lowest level of the universe, that is, planets. Regardless of the level, all galaxies exist in the form of a sphere consisting of many secondary galaxies and their orbits, and this sphere moves regularly in a similar circular elliptical orbit. The kinetic energy and potential energy are the existing basic forms of energy in the universe, and the internal energy is a complementary existing form of energy under relativity rest condition. Because of the multiple motion of the universe, the kinetic energy in the universe exists on the division of energy levels, the higher the levels of the galaxy structure is, the lower the kinetic energy levels is, and the lower the levels of a galaxy structure is, the higher its kinetic energy levels is. Although there is an evolution of mass and energy distribution in the universal system, but it is eternal for the whole universal system.

\section{DISCUSSION}

materialism world view in the discussion of the universe

Dialectical materialism holds the world is essentially material. Engels said, "The real unity of the world lies in its materiality." The material world moves, changes, and develops according to its inherent laws. Material is primary and the consciousness is secondary. The universe is made up of objective matter, if we deny the objectivity of the matter, energy, and space to discuss the problem of the universe, then we can only fall into the quagmire of the idealism and never get the correct conclusion. In fact, although there is the evolution of mass and energy's distribution in the universal system, it is eternal for the whole universal system. Denying the objectivity of existence of matter, the primacy of matter, and the eternity of the universe, it can only make us deviate from the dialectical materialist worldview and produce a bias for understanding of the objective material world. It can be said the highest level of understanding of the universe does not stop in the field of physics, but extends to the field of philosophy. The problem of world view is not only the fundamental point, but also is a starting point for us to understand the objective material world.

\section{REFERENCE}

[1] Chen Gong fu. New Exploration of the Mystery of the Universe. (Jilin People's Publishing House2000), P22

[2] http://coolcosmos.ipac.caltech.edu/page/ galactic_center

[3] https://baike.baidu.com/item/\%E9\%93\% B6\%E5\%BF\%83/948866? fr=aladdin

[4] Wu Yue-ming, 2016, Study on the Function of Jupiter in the Formation of Sunspots Weeks, Astronomy and Astrophysics.

http://dx.doi.org/10.12677/AAS.2016.41001

[5] Reidy, Michael S, 2017, The Most Recent Orogeny, ResearchGate,

DOI : 10.1525/hsns.2017.47.4.578

[6] Zhang Mingzhen, etc., 2018, Mid-Cretaceous Hothouse Climate and the Expansion of Early Angiosperms, ACTA GEOLOGICA SINICAENGLISH EDITION.

DOI 10.1111/1755-6724.13692 